\title{
Percepción de habilidad, reto y relevancia como predictores de compromiso cognitivo y afectivo en estudiantes de secundaria*
}

\section{Perception of Ability, Challenge and Relevance as Predictors of Cognitive and Affective Engagement in High School Students}

Recepción: 01 Junio 2018 | Aceptación: 06 Julio 2018

\author{
Solanlly OchoA-Angrino ${ }^{\mathrm{a}}$ \\ Pontificia Universidad Javeriana de Cali, Colombia \\ ORCID: http://orcid.org/0000-0002-7043-2808 \\ Jairo A. Montes-GonzÁlez \\ Pontificia Universidad Javeriana de Cali, Colombia \\ ORCID: http://orcid.org/0000-0003-1778-1096 \\ Tatiana Rojas-Ospina \\ Pontificia Universidad Javeriana de Cali, Colombia \\ ORCID: http://orcid.org/0000-0003-0212-8537
}

\footnotetext{
${ }^{a}$ Autor de correspondencia. Correo electrónico: sochoa@javerianacali.edu.co

Para citar este artículo: Ochoa-Angrino, S., MontesGonzález, J. A., \& Rojas-Ospina, T. (2018). Percepción de habilidad, reto y relevancia como predictores de compromiso cognitivo y afectivo en estudiantes de secundaria. Universitas Psychologica, 17(5), 1-18. http://doi.org/10.11144/Javeriana.upsy17 -5 .phrr
}

\section{RESUMEN}

Este estudio evalúa la relación entre percepción de habilidad, reto, relevancia de la tarea y compromiso cognitivo y afectivo en 88 estudiantes colombianos de secundaria durante clases de Ciencias. Igualmente, indaga si las relaciones entre percepción de habilidad, reto, relevancia y compromiso están moderadas por tipo de asignatura y género, y si existe relación entre compromiso y desempeño académico. El diseño del estudio es descriptivo correlacional y predictivo. Se usó el método de muestreo de experiencias (ESM) para tomar medidas repetidas del compromiso y de las percepciones de habilidad, reto y relevancia. Dada la característica anidada de los datos, se realizaron análisis lineales jerárquicos. Las percepciones de habilidad, reto y relevancia predicen el compromiso cognitivo y afectivo. Género y tipo de curso emergen como moderadores de las relaciones entre estas percepciones y compromiso. A su vez, el compromiso cognitivo y afectivo predice el desempeño académico en Ciencias. Se discuten las implicaciones educativas del estudio.

Palabras clave

compromiso cognitivo y afectivo; percepción de reto; percepción de habilidad; percepción de relevancia; desempeño académico.

\begin{abstract}
This study evaluates the relationship between perception of ability, challenge, relevance of the task, and cognitive and affective engagement in 88 Colombian high school students during science classes. Likewise, it investigates if the variables: type of subject and gender; moderate the relationships between perception of ability, challenge, relevance, and engagement. Moreover, if there is a relationship between engagement and academic performance. The design of the study is descriptive, correlational and predictive. The experience sampling (ESM) method was used to take repeated measures of engagement and perceptions of ability, challenge, and relevance. Given the nested characteristics of the
\end{abstract}


data, linear hierarchical analyzes were performed. Perceptions of ability, challenge and relevance predict cognitive and affective engagement. Gender and type of course emerge as moderators of the relationships between perception of challenge, ability, relevance and engagement. In turn, cognitive and affective engagement predicts academic performance in science. The educational implications of the study are discussed.

Keywords

cognitive and affective engagement; perception of challenge; perception of ability; perception of relevance; academic performance.

El compromiso de los estudiantes en contextos académicos implica involucramiento cognitivo, emocional y comportamental de los aprendices en actividades escolares y extraescolares (Skinner, Kindermann, \& Furrer, 2009). El compromiso cognitivo ha sido definido como el interés, la concentración, el esfuerzo consciente por relacionar conocimiento nuevo con previo, encontrar usos al conocimiento, organizar personalmente el saber, buscar activamente ejemplos para ayudarse a entender y relacionar conocimiento entre cursos académicos, entre otros (Lam et al., 2014; Shernoff \& Bempechat, 2014; Strati, Schmidt, \& Maier, 2017). El compromiso afectivo se caracteriza por entusiasmo, disfrute, ánimo, gusto por las actividades escolares, orgullo de ser estudiante, satisfacción en la escuela, vitalidad y felicidad durante las jornadas escolares (Hill \& Werner, 2006; Lam et al., 2014; Shernoff \& Bempechat, 2014). El compromiso comportamental se relaciona con la persistencia, el esfuerzo y la intensidad del mismo para realizar tareas académicas (Skinner \& Belmont, 1993).

Numerosos investigadores han señalado los beneficios del compromiso cognitivo y afectivo para el aprendizaje significativo y para el óptimo desempeño en diferentes áreas. Csikszentmihalyi, Abuhamdeh y Nakamura (2005) y Nakamura (1988) encontraron que los estudiantes que mostraron mayor compromiso en Matemáticas obtuvieron mejores desempeños en las pruebas correspondientes; en escritura, Larson (1988) reporta que los estudiantes más comprometidos con las tareas de un ensayo escribieron textos más largos, más complejos y completos y estaban dispuestos a revisarlos varias veces; en procesos de lectura, Ochoa-Angrino (2012) encontró que los estudiantes de secundaria con mayores niveles de compromiso durante la lectura de textos en clases de Ciencias tuvieron mejores calificaciones que sus pares menos comprometidos; resultados similares se han encontrado en creatividad artística y científica (Csikszentmihalyi, 2008; Nakamura \& Csikszentmihalyi, 2002). En un estudio reciente realizado en 12 países, Lam et al. (2014) hallaron relaciones significativas positivas entre desempeño académico y compromiso cognitivo, afectivo y comportamental.

El compromiso se ha asociado positivamente con el bienestar psicológico de los estudiantes; es decir, los más comprometidos muestran alta autoestima, buenos niveles de responsabilidad académica, optimismo y esperanza, y presentan menos ausentismo que sus pares poco comprometidos; por otra parte, se han encontrado relaciones entre la falta de compromiso académico y ausentismo escolar, pérdida de cursos y deserción académica (Furrer, Skinner, \& Pitzer, 2014; Steinberg, Brown, \& Dombush, 1996).

Dada la relevancia del compromiso para el aprendizaje y para el desempeño académico, diversos estudiosos se han enfocado en indagar los factores intrapersonales, interpersonales y contextuales que lo promueven o lo inhiben. $\mathrm{Al}$ respecto, se encuentran investigaciones que hacen énfasis en estudiar cómo el compromiso se ve influido por factores externos tales como la relevancia del medio ambiente escolar y el clima del salón de clases (Patrick, Ryan, \& Kaplan, 2007; Reyes, Brackett, Rivers, White, \& Salovey, 2012; Shukla, Konold, \& Cornell, 2015; Skinner, Furrer, Marchand, \& Kindermann, 2008). Otros estudios se centran en analizar el papel de las variables individuales y su relación con el compromiso, por ejemplo, estudian cómo las percepciones que los estudiantes tienen con respecto a ellos mismos como aprendices (sus habilidades, la relevancia que le atribuyen a las tareas, el interés que les generan) y el nivel de reto que demandan, influyen en los niveles de compromiso académico (Ochoa-Angrino, 2012; Shernoff, 2012; Shernoff \& Anderson, 2013; 
Shernoff \& Bempechat, 2014). Por último, otros investigadores se han centrado en estudiar la relación entre variables intrapersonales y contextuales, por ejemplo, la relación con el compromiso y las percepciones de los estudiantes con respecto al reto que implican las tareas académicas y el apoyo que ofrecen los docentes (Strati et al., 2017).

Relación entre compromiso y percepción de reto y habilidad

Uno de los factores al que se le ha otorgado mayor relevancia en el estudio del compromiso cognitivo y afectivo es el balance entre las percepciones sobre el nivel de reto que demanda una tarea y el nivel de habilidad que se tiene para resolverla. Este factor parte de los estudios de Csikszentmihalyi y Csikszentmihalyi (1988), Csikszentmihalyi et al. (2005), Csikszentmihalyi (2008) y, recientemente, los estudios de Shernoff, Abdi, Anderson y Csikszentmihalyi (2014). Según estos autores el compromiso se logra cuando el reto que suponen las tareas académicas está en balance con las habilidades del estudiante y este experimenta cierto control sobre los procesos de aprendizaje.

En este sentido, los estudiantes deben percibir que las tareas académicas les proveen un nivel de exigencia entre moderado y alto, de tal manera que desafíen pero no rebasen las habilidades existentes. Específicamente, los adolescentes prefieren tareas retadoras que les demanden algún tipo de esfuerzo extra en comparación con tareas que son demasiado fáciles (Fullagar Knight, \& Sovern 2013; Nakamura \& Csikszentmihalyi, 2002; Shumow \& Schmidt, 2013). Cuando los estudiantes identifican mayor reto, ellos reportan más esfuerzo, interés y concentración (Shernoff, 2012; Shernoff, Csikzentmihalyi, Schneider, \& Shernoff, 2003; Shernoff \& Schmidt, 2008). Cuando las mujeres perciben que la actividad es retadora tienden a reportar menor compromiso cognitivo y afectivo y más baja autoestima, en comparación con los hombres (Schmidt, Strati, \& Kackar, 2010; Schmidt \& Smith, 2008).
Strati et al. (2017) señalan que factores como el estereotipo de género sobre el aprendizaje de las Ciencias podría estar relacionado con estos resultados. Por su parte, la percepción de las habilidades entra en interacción con el esfuerzo que hacen los estudiantes, sus estrategias de estudio, sus logros académicos y posteriores elecciones vocacionales (Shumow \& Schmidt, 2014).

Cuando no se percibe equilibrio entre reto y habilidad, el compromiso con el aprendizaje decae y pueden emerger aburrimiento, ansiedad o apatía. El aburrimiento surge cuando las habilidades son altas y el reto bajo; la ansiedad, cuando las habilidades son escasas y el reto alto y la apatía, cuando reto y habilidad se perciben en nivel bajo (Csikszentmihalyi \& Csikszentmihalyi ,1988). En síntesis, la percepción de reto y habilidad en un nivel alto es una condición importante para facilitar experiencias óptimas como el compromiso cognitivo y afectivo.

Percepción de relevancia personal de la tarea y compromiso cognitivo y afectivo

Otro de los aspectos centrales en la promoción del compromiso cognitivo y afectivo tiene que ver con la percepción de relevancia de las tareas. Ante cada tarea académica, los estudiantes evalúan qué tanta relación tiene con su vida, con sus metas académicas y no académicas, con sus intereses personales y con las actividades que realizan fuera de la escuela (Canning \& Harackiewicz, 2015; Rozek, Hyde, Svoboda, Hulleman, \& Harackiewicz, 2015). Estos autores plantean que los estudiantes no dedican tiempo ni esfuerzo para aprender en actividades académicas que carecen de relevancia personal; es decir, aquellas actividades escolares que no guardan relación con sus necesidades e intereses fuera del salón de clase. En estudios sobre la percepción de relevancia en tareas de lectura, Smith y Wilhelm (2002) encontraron que estudiantes de secundaria quienes no leían los textos de Ciencias que proponían los profesores y eran reportados en la 
escuela como malos lectores, en casa mostraban todo lo contrario. Eran asiduos lectores de revistas de mecánica automotriz y basados en textos y en su comprensión de los mismos emprendían tareas complejas relacionadas con la reparación de sus autos. Los autores concluyen que la relevancia personal es un factor que deben tener en cuenta los educadores a la hora de diseñar actividades de lectura.

Hulleman, Godes, Hendricks y Harackiewicz (2010) y Vansteenkiste, Simons, Lens, Sheldon y Deci (2004) reportan relaciones significativas entre percepción de relevancia y desempeño en Ciencias, mientras que Koballa y Glynn (2007) encuentran relaciones significativas entre percepción de relevancia y persistencia en las tareas de Ciencias.

A pesar de la importancia que el compromiso ha demostrado tener con la participación activa en las tareas propias de vida escolar y su relación con el aprendizaje y el desempeño en diferentes áreas, muchos estudios sobre el compromiso se han centrado en indagar cómo se da en actividades extraescolares (Shernoff, 2010; Shernoff \& Vandell, 2007) o basan sus estudios en preguntas generales sobre el recuerdo de compromiso en la escuela; por ejemplo, usando ítems que invitan a los estudiantes a dar un informe general frente al estudio: "Cuando yo estudio trato de relacionar el material para entenderlo mejor" (Lam et al., 2014; Wan, Bergin, \& Bergin, 2014). Solo recientemente han aumentado las investigaciones que se han enfocado en estudiar el compromiso cognitivo y afectivo en el salón de clases, en el momento en que los estudiantes participan de las actividades (Ochoa-Angrino, 2012; Schmidt, Shumow, \& Zaleski, 2012; Schmidt, Rosenberg, \& Beymer, 2017; Shernoff et al., 2016; Strati et al., 2017).

Las medidas del compromiso cognitivo y afectivo basadas en el recuerdo de lo que ocurrió mientras se participaba en la clase, pese a ser valiosas, tienen el riesgo de ser incompletas. Cuando los investigadores indagan a los estudiantes mucho tiempo después de que ocurren los eventos de aprendizaje, los estudiantes tienden a recordar con inexactitud su experiencia y a escoger respuestas socialmente deseables que no se corresponden con lo que realmente sucedió en el evento de aprendizaje (Fleeson, 2007; Zuzanek, 1999). La necesidad de caracterizar el compromiso cognitivo y afectivo en tiempo real a partir de medidas repetidas, es todavía más pertinente si se tiene en cuenta que puede variar a lo largo de una tarea, de diferentes tareas, de una sesión de clase o de una unidad temática (Hektner, Schmidt, \& Csikszentmihalyi, 2007; Vitiello, Booren, Downer, \& Williford, 2012). Retomando la teoría de la motivación emergente, Strati et al. (2017) plantean que el compromiso debe ser estudiado como algo maleable, que puede emerger o no de acuerdo con patrones contextuales específicos y de las percepciones de los estudiantes frente a estos, más que como una característica estable de la personalidad (por ejemplo, cuando se señala que un estudiante es en general comprometido con el aprendizaje).

En el caso de Colombia y en general de Latinoamérica, los estudios sobre compromiso cognitivo y afectivo en contextos educativos y sobre los factores que lo promueven son casi inexistentes. Uno de los pocos estudios fue el realizado por Rigo (2017), donde relaciona el compromiso cognitivo, afectivo y comportamental con las características de las interacciones profesor-alumno en el salón de clases, en cursos de Ciencias Sociales. Participaron cuatro profesores y 83 estudiantes de educación primaria de Argentina. Se realizaron observaciones del contexto de enseñanza y aprendizaje y se aplicaron cuestionarios que indagaban por el compromiso cognitivo y afectivo de los alumnos. Se encuentran relaciones significativas entre el compromiso de los estudiantes y las características del contexto de enseñanza. Prácticas de enseñanza que favorecieron la frecuencia y la calidad de la retroalimentación, oportunidades para aprender, participación de los educandos en la toma de decisiones sobre aspectos de la clase, entre otras, estuvieron positivamente relacionadas con su compromiso cognitivo, afectivo y comportamental.

El presente estudio aporta en tres sentidos: (a) contribuye al campo de estudio sobre variables 
individuales asociadas al compromiso cognitivo y afectivo; (b) estudia dicho compromiso en el salón de clases, tomando en cuenta lo que sucede en tiempo real en dicho escenario y (c) aporta datos de estudiantes colombianos en clases de Ciencias Naturales.

Basado en la revisión de la literatura, este estudio evalúa cuatro hipótesis:

Hipótesis 1: Las percepciones que tienen los estudiantes sobre el reto que les demandan las tareas académicas, el nivel de habilidad con que cuentan para realizarlas y la relevancia que otorgan a las mismas están relacionadas positivamente con el compromiso cognitivo y afectivo que experimenten durante las clases de Ciencias.

Hipótesis 2: El nivel de compromiso cognitivo y afectivo varía de acuerdo al género y al tipo de asignatura.

Hipótesis 3: Las relaciones positivas entre reto, habilidad y compromiso cognitivo y afectivo están moderadas por el género y el tipo de asignatura.

Hipótesis 4: El compromiso cognitivo y afectivo predice el rendimiento académico en Ciencias.

\section{Método}

Tipo de estudio

Descriptivo, correlacional y predictivo con análisis basados en medidas repetidas.

\section{Participantes}

Participaron voluntariamente, previo consentimiento y asentimiento firmado, 88 estudiantes colombianos de secundaria de dos escuelas públicas del municipio de Palmira (Valle). La edad promedio de los participantes fue de 16 años $(M E=16, D E=1.47)$; el $57 \%$ fueron mujeres; el $55.6 \%$ estaban matriculados en grado $9^{\circ}$, el $17 \%$ en $10^{\circ}$, y el $27.2 \%$ en $11^{\circ}$. Los estudiantes estaban distribuidos en los siguientes cursos: Educación Ambiental (grado 9o, 26 \%),
Biología (grado 9o 29 \%), Química (grado 10º 17 \%) y Física (grado 11ํㅡㄹ 27 \%).

\section{Instrumentos}

La experiencia subjetiva de los estudiantes sobre el compromiso cognitivo y afectivo en el salón de clase, se evaluó utilizando el método de muestreo de experiencias (MME) (Csikszentmihalyi \& Larson, 1987; Zuzanek, 1999), que consiste en tomar muestras de los reportes de las experiencias subjetivas tales como percepción de éxito, bienestar, concentración y disfrute, cuando las personas están realizando diferentes actividades como leer, escribir, escuchar una presentación, etc. El MME tiene alta validez ecológica, pues captura las diferentes percepciones en tareas que las personas realizan en su vida cotidiana; tiene alta validez interna porque, al demandar respuestas inmediatas, reduce fallos atribuibles a la memoria, frecuentes en cuestionarios que indagan por este tipo de experiencias una vez terminada una actividad o mucho después de su realización, y reduce la posibilidad de respuestas socialmente deseables (Hektner et al., 2007). El método de muestreo de las experiencias fue utilizado a lo largo de una unidad temática escogida por los profesores de Ciencias. Las unidades temáticas tomaron entre 3 y 6 clases.

\section{Cuestionario de Motivación Emergente (CME)}

El CME usado en esta investigación es una adaptación del instrumento usado por Schmidt y Smith (2008). Se compone de 24 ítems que examinan los estados emocionales y cognitivos de los estudiantes durante la realización de actividades académicas en el salón de clases. El compromiso cognitivo y afectivo incluye ítems como: "¿Qué tan concentrado estabas?", "iestabas teniendo éxito en la tarea?". La dimensión afectiva incluye ítems como "iestabas disfrutando de lo que estabas haciendo?", “iTe sentías bien contigo mismo en ese momento?". El CME se califica con una escala tipo Likert, rango de 1 a 5 , donde 1 significa nada y 5 significa mucho. En Colombia, no se reportan usos previos 
del cuestionario. Para este estudio, el CME fue traducido del inglés al español y esta traducción se volvió a traducir al inglés por un profesor nativo, para controlar que el vocabulario usado en el cuestionario fuera adecuado al contexto colombiano sin traicionar el objetivo del CME. Se usaron los programas estadísticos IBM SPSS v.23 y IBM SPSS Amos 24.0.0, para la realización del Análisis Exploratorio de Factores y el Análisis Confirmatorio de Factores, respectivamente. En principio, los 24 ítems del instrumento se sometieron a un análisis exploratorio de factores con rotación oblicua en una muestra de 116 estudiantes de los grados $9^{\circ}$ a $11^{\circ}$. Las puntuaciones del instrumento cumplieron con el supuesto de normalidad univariada. Por su parte, el coeficiente de curtosis normalizada multivariado de Mardia fue de 34.301 para todos los ítems, indicando la presencia de no normalidad multivariada en la distribución de las puntuaciones. Posteriormente, se evaluaron las características de las matrices: el test de esfericidad de Bartlett $\left(\chi^{2}=5265.074, g l=435, p\right.$ $<0)$ fue significativo, la medida de Kaiser-MeyerOlkin de adecuación de la muestra fue $=0.913$ y las Medidas Individuales de la Adecuación de la Muestra (MSA) fueron superiores a 0.8 en todos los ítems, indicando que la muestra era suficiente y que la matriz de correlaciones es factorizable. Se examinó también la tabla de comunalidades, y se eliminaron cinco ítems que presentaron bajas comunalidades $(>0.4)$. Con el fin de identificar los factores por extraer, se consideró el test MAP revisado (Minum Average Partial Test) propuesto por Velicer, Eaton y Fava (2000); el test AP (Parallel Analysis) y la significancia teórica de los factores (Lloret-Segura, Ferreres-Traver, Hernández-Baeza, \& Tomás-Marco, 2014). Se usaron los siguientes criterios con el fin de determinar los factores y los ítems que estarían en la solución final: (1) al menos tres ítems con cargas altas en la matriz de patrones para identificar un factor (Lloret-Segura et al., 2014); (2) se retuvieron ítems con coeficientes de patrón iguales o superiores a 0.4 en la matriz de factores y de patrones. Siguiendo estos criterios, se extrajeron dos factores: Compromiso cognitivo y afectivo que explica el $36.68 \%$ de la varianza
(13 ítems) y Afecto positivo y negativo que explica el $8.26 \%$ de la varianza (5 ítems).

Luego se realizó el Análisis Confirmatorio de Factores con base en la estructura factorial arrojada por el análisis previo y con una nueva muestra de 103 estudiantes de grados noveno a once. Se consideró el estadístico chi-cuadrado que prueba el modelo nulo frente al propuesto y se incorporó la diferencia entre el $\chi^{2} / g l$, la cual se considera un buen indicador si el resultado oscila entre 1 y 3 (Carmines \& Mclver, 1981); también se incluyó el error cuadrático medio de aproximación (RMSEA), el cual, de acuerdo con McDonald y Ho (2002), indica un buen ajuste cuando presenta valores inferiores a 0.05 y un ajuste aceptable cuando presenta valores inferiores a 0.08. Además, se consideró la medida de ajuste comparativo (CFI), donde valores cercanos a 0.9 son considerados aceptables (Netemeyer, Bearden, \& Sharma, 2003). Al hacer el análisis, fue necesario eliminar un ítem del factor Compromiso cognitivo y afectivo y dos del factor Afecto positivo y negativo; los ítems eliminados correspondían a reactivos relacionados con el afecto negativo, así que este factor finalmente pasó a denominarse Afecto positivo. También se correlacionaron los residuos de los ítems 4 y 23, 6 y 9, y 11 y 13 del factor Compromiso cognitivo y afectivo y los residuos de los ítems 15 y 17 del factor Afecto positivo. Una vez realizados estos ajustes, el modelo de dos factores presentó un ajuste aceptable a los datos $\left(\chi^{2}=132.03, \mathrm{p}=0.001 ; \chi^{2} / g l=1.553 ;\right.$ $\mathrm{RMSEA}=0.074 ; \mathrm{CFI}=0.911)$. Posteriormente, se evaluó la confiabilidad de las dos escalas por medio del alpha de Cronbach, encontrando valores buenos $(\mathrm{a}>0.8)$.

\section{Medidas}

\section{Variables dependientes}

Compromiso Cognitivo y Afectivo: representa el nivel en el que los estudiantes se involucran cognitiva y afectivamente en las clases de Ciencias, evaluado mediante el promedio de cuatro ítems del CME: disfrute (¿Estabas 
disfrutando de lo que estabas haciendo?), concentración (¿Qué tan concentrado estabas durante la clase de Ciencias?), bienestar (iTe sentías bien contigo mismo en ese momento?) y éxito (iEstabas teniendo éxito en lo que estabas haciendo?).

Desempeño académico. Calificaciones finales del curso, rango 0-5, donde cero indica el nivel más bajo y cinco el nivel más alto de desempeño.

\section{Variables predictoras}

Tres ítems fueron tomados del CME, de manera independiente: (1) Percepción de habilidad (iQué tan hábil te sentías realizando esta actividad?) durante las clases de Ciencias, (2) Percepción de reto (¿Qué tan retadora era la actividad?) y (3) Percepción de relevancia (iEsta actividad era importante para ti?).

\section{Variables moderadoras}

(1) Género, reportado por los estudiantes en un formato de datos sociodemográficos; (2) Asignatura, los participantes en el estudio fueron observados en clases de Educación Ambiental ( $9^{\circ}$ grado), Biología ( $9^{\circ}$ grado diferentes a los de Educación Ambiental), Química (10ำ grado) y Física (11ํo grado).

\section{Procedimiento}

Previamente a la recolección de datos, los investigadores explicaron la manera de diligenciar el CME a los estudiantes que aceptaron participar voluntariamente en la investigación. Además, se llevó a cabo una sesión previa de familiarización con los instrumentos durante una clase regular. Durante la recolección de datos, los alumnos fueron llamados, a través de una alarma de celular, a responder al CME dos (2) veces durante cada clase a lo largo de la unidad temática estudiada. Para evitar disrupciones y tomar registro de una mayor variedad de actividades en las clases, los participantes de cada grupo fueron divididos por mitad, y cada subgrupo respondió en momentos diferentes de la clase. Al final de la recolección de datos, cada estudiante había diligenciado en promedio ocho veces el CME, para un total de 667 reportes.

\section{Análisis de la información}

A través del software Hierarchical Linear and Nonlinear Modeling ([HLM 7]; Raudenbush, Bryk, \& Congdon, 2010), se usaron modelos de análisis cuantitativos, específicamente modelamiento lineal jerárquico por la característica anidada de los datos (Percepción de habilidad, Percepción de reto y Percepción de relevancia al interior de los estudiantes, quienes a su vez hicieron parte de grupos según asignaturas y género) (Raudenbush \& Bryk, 2002). El primer modelo utilizado es un modelo nulo que representa el valor promedio del compromiso cognitivo y afectivo sin la presencia de las variables predictoras, que pudieran explicar su variación. Este modelo informa si el Compromiso cognitivo y afectivo es significativamente variable como para justificar análisis posteriores con la inclusión de factores, en la forma de un modelo condicional (véase Apéndice 1). Se utilizaron modelos condicionados para evaluar si las percepciones momentáneas de los estudiantes acerca de sus habilidades en las clases, el reto que les representaban las tareas y la relevancia que percibían respecto de las mismas, estaban relacionados con el compromiso (Variables de nivel I), al igual que para analizar posibles variaciones en estas percepciones de acuerdo a género y asignatura (Variables de nivel II). En el Apéndice 1 se ejemplifica un modelo condicionado de los que se emplearon. Finalmente, se utiliza un análisis de regresión lineal bivariante para examinar la relación entre el compromiso y el desempeño académico en Ciencias. 


\section{Resultados}

\section{Resultados generales}

Las medidas presentaron una distribución normal y tendieron a ubicarse en puntajes medios; aunque hay relaciones significativas entre las medidas estas no son altas, lo que indica que no hay problemas de multicolinearidad (Leech, Barrett, \& Morgan, 2008) (Tabla 1).

\section{Tabla 1}

Estadísticas descriptivas y correlaciones de las medidas $(r)$

\begin{tabular}{lrrrrrrr}
\hline \multicolumn{1}{c}{ Medidas } & Media & DE & Compromiso & Habilidad & Relevancia & \multicolumn{1}{c}{ Reto } & $\begin{array}{c}\text { Desempeño } \\
\text { académico }\end{array}$ \\
\hline Compromiso & 3.27 & 0.94 & 1 & $0.644^{*}$ & $0.591^{*}$ & $0.43^{*}$ & $0.161^{*}$ \\
Habilidad & 3.08 & 1.1 & $0.644^{*}$ & 1 & $0.496^{*}$ & $0.422^{*}$ & $0.122^{*}$ \\
Relevancia & 3.38 & 1.14 & $0.591^{*}$ & $0.496^{*}$ & 1 & $0.47^{*}$ & $0.225^{*}$ \\
Reto & 2.72 & 1.2 & $0.43^{*}$ & $0.422^{*}$ & $0.47^{*}$ & 1 & $0.207^{*}$ \\
$\begin{array}{l}\text { Desempeño } \\
\text { académico }\end{array}$ & 3.61 & 0.68 & $0.161^{*}$ & $0.122^{*}$ & $0.225^{*}$ & $0.207^{*}$ & 1 \\
\hline
\end{tabular}

Nota. $N=88$ estudiantes, 670 respuestas, ${ }^{*} p$ $<0.001$. Escala de calificación de compromiso, habilidad, relevancia y reto de 1-5, 1: nada, 2: un poco, 3: algo, 4: bastante y 5: mucho. Desempeño académico: rango $0-5$, donde cero corresponde al desempeño más bajo y 5 al más alto.

Los estudiantes reportaron algo de compromiso cognitivo y afectivo durante las clases de Ciencias, percibieron poco reto, algo de habilidad y algo de relevancia. Al observar los desempeños generales por curso, se aprecia que los estudiantes de Biología y Física tienden a considerar que son algo hábiles para las actividades de Ciencias, mientras que los de Educación Ambiental y Química perciben poca habilidad. Los alumnos perciben poco reto en las clases, con excepción de los de Física quienes perciben que las actividades son algo retadoras. La relevancia atribuida a las actividades de la clase es moderada (algo importantes), tendiendo a percibirse como poco relevantes en los estudiantes de Biología y bastante relevantes en los estudiantes de Física. Estos últimos también reportan los puntajes más altos en todas las medidas, mientras que los de Educación Ambiental y Biología presentaron los puntajes más bajos (véanse detalles en la Tabla 2).
Tabla 2

Medias y desviaciones estándar del compromiso cognitivo y afectivo, el reto, la habilidad, la relevancia y el desempeño por asignatura

\begin{tabular}{lcrrr}
\hline \multirow{2}{*}{ Variables } & \multicolumn{1}{c}{ Educación Ambiental } & \multicolumn{1}{c}{ Biología } & Química & \multicolumn{1}{c}{ Física } \\
\cline { 2 - 5 } & Media $(D E)$ & Media $(D E)$ & Media $(D E)$ & Media $(D E)$ \\
\hline Compromiso & $3.22(0.57)$ & $3.08(0.66)$ & $3.03(0.35)$ & $3.54(0.86)$ \\
Habilidad & $2.95(0.72)$ & $3.13(0.77)$ & $2.95(0.35)$ & $3.24(0.9)$ \\
Reto & $2.23(0.64)$ & $2.71(0.01)$ & $2.68(0.68)$ & $3.37(0.89)$ \\
Relevancia & $3.32(0.86)$ & $2.97(0.9)$ & $3.29(0.75)$ & $3.74(0.92)$ \\
Desempeño & $3.33(0.94)$ & $3.35(0.94)$ & $3.92(0.69)$ & $3.8(0.62)$ \\
\hline
\end{tabular}

Nota. Valores de las variables dependientes e independientes: Mínimo 1 y Máximo 5.

\section{Resultados modelo nulo}

El único efecto fijo estimado fue el intercepto -compromiso cognitivo y afectivo-, $\gamma_{00}$. El promedio de compromiso durante las clases de Ciencias fue de $3.25(D E=0.07)$. Este coeficiente difiere significativamente de $0(t(88)$ $=44.627, p<0.001) \mathrm{e}$ indica que hay suficiente variabilidad en el compromiso de los estudiantes durante las clases de esta asignatura. La proporción de la variabilidad de compromiso durante las clases de Ciencias se calculó usando la correlación intraclase $(\mathrm{CCI}=0.3965 / 0.51409$ $+0.3965=0.4354)$, indicando que $43.54 \%$ de la variabilidad en compromiso es intraindividual mientras que el $56.46 \%$ es interindividual. Estos resultados señalan la pertinencia de incluir predictores para ver en qué medida ayudan a explicar la variabilidad del compromiso cognitivo y afectivo.

Se usaron modelos lineales jerárquicos condicionales para evaluar la Hipótesis 1, es decir, para evaluar si había relaciones significativas positivas entre compromiso cognitivo y afectivo y las percepciones de los estudiantes en relación con sus habilidades para realizar las tareas, el reto que les implicaban y la relevancia que les otorgaban. Se utilizó un primer modelo condicional con predictores de Nivel I. La percepción de reto resultó positivamente asociada con el compromiso $\left(\gamma_{10}\right.$ $=0.09, p<0.05)$. Esto quiere decir que cuando las actividades académicas de Ciencias son percibidas como retadoras los estudiantes tienden a experimentar mayor compromiso, y que cada punto en la percepción de reto aporta 0.09 
al promedio de compromiso. La percepción de habilidad también estuvo positivamente asociada con el compromiso $\left(\gamma_{20}=0.32, p<0.001\right)$, es decir, en la medida en que los estudiantes se perciben competentes su compromiso aumenta; cada punto en la percepción de habilidad aporta 0.32 al promedio. Igualmente, la percepción de relevancia de la tarea se relacionó positivamente con el compromiso $\left(\gamma_{30}=0.23, p<0.001\right)$. Es decir, a mayor relevancia percibida en las actividades de Ciencias, mayor compromiso cognitivo y afectivo por parte de los estudiantes. La percepción de relevancia aporta 0.23 al promedio del compromiso. El $45.75 \%$ de la varianza del compromiso se explica por la percepción de reto, habilidad y relevancia $(0.51409-0.27887 / 0.51409=0.4575)$. La Tabla 3 resume los resultados.

Un segundo modelo condicionado se usó para evaluar las segunda y tercera hipótesis de investigación, es decir, para indagar si el compromiso era igual o diferente para los estudiantes de acuerdo al tipo de asignatura y género, y si estos modulaban las relaciones entre el compromiso y las percepciones de reto, habilidad y relevancia antes descritas. Para realizar dicho análisis, se crearon dos variables indicadoras, una por asignatura y otra por género 1 . En este modelo, el intercepto representa el promedio de compromiso cognitivo y afectivo de los estudiantes de Física en comparación con los estudiantes de Química, Biología y Educación Ambiental cuando se controlan reto, habilidad y relevancia $\gamma_{00}=3.36$. No hay coeficientes significativos para género, lo cual indica que hombres y mujeres experimentan niveles similares de compromiso en las clases de Ciencias cuando se controla la percepción de reto, habilidad y relevancia. En relación con el tipo de curso, el nivel de compromiso de los estudiantes de Física $\left(\gamma_{00}=3.36, p<\right.$ 0.001) es significativamente mayor que el de los estudiantes de química $\left(\gamma_{04}=-0.23, p<0.05\right)$, lo cual indica que un estudiante de Química tendrá 0.23 menos puntaje en el promedio de compromiso cognitivo y afectivo que uno de Física. El género modula las relaciones entre la percepción de reto y compromiso. Mientras la relación entre compromiso y género tiende a ser positiva para los hombres $\left(\gamma_{10}=0.12\right.$, $p<0.1)$, esta relación se invierte totalmente para las mujeres $\left(\gamma_{11}=-0.12, p<0.05\right)$. Esto quiere decir que los hombres experimentan mayor incremento en el compromiso cognitivo y afectivo cuando perciben que las actividades en el salón de clase son retadoras, comparados con sus compañeras. El tipo de asignatura no modula las relaciones entre la percepción de reto y el compromiso.

El género no modula la relación entre percepción de habilidad y compromiso, mientras que el tipo de asignatura sí modula la relación entre percepción de habilidad y compromiso cognitivo y afectivo, en algunos cursos. Los resultados muestran diferencias significativas entre los estudiantes del curso de Física y los de Educación Ambiental y Biología. La fuerza de la asociación entre compromiso y habilidad es mayor en los estudiantes de Física $\left(\gamma_{20}=0.49\right.$ $p<0.001)$ comparados con los estudiantes de Educación Ambiental $\left(\gamma_{22}=-0.26, p<0.05\right)$ y Biología $\left(\gamma_{23}=-0.26, p<0.05\right)$. Es decir que los estudiantes de Física comparados con los de Educación Ambiental y Biología experimentan casi el doble de incremento en compromiso, cuando se perciben hábiles en la realización de las tareas. No se encontraron diferencias significativas por género ni por asignatura en la relación entre compromiso académico y relevancia.

El Nivel 1 de varianza en el compromiso explicada por la inclusión de predictores de Nivel II, asignatura y género, no se reduce. El nivel de varianza del intercepto se reduce de 0.0997 del modelo condicional 1 a 0.08262 (0.0997 $0.08262 / 0.0997=0.1713)$; es decir que el 17.13 $\%$ de la variancia del compromiso se explica por la inclusión de predictores de Nivel II. La Tabla 3 resume los resultados. 
Tabla 3

Relaciones entre compromiso cognitivo y afectivo $y$ percepciones de reto, habilidad, relevancia y asignatura y género como moderadores de dichas relaciones

\begin{tabular}{|c|c|c|c|c|}
\hline & \multicolumn{2}{|c|}{ Modelo 1} & \multicolumn{2}{|c|}{ Modelo 2} \\
\hline Efectos fijos & $B$ & $S E$ & $B$ & $S E$ \\
\hline Intercepto, $\gamma_{00}$ & $3.28 * *$ & 0.04 & $3.36^{* *}$ & 0.1 \\
\hline Género, $\gamma_{01}$ & & & 0.004 & 0.08 \\
\hline Educación Ambiental, $\gamma_{02}$ & & & -0.1 & 0.12 \\
\hline Biologia, $\gamma_{03}$ & & & -0.1 & 0.13 \\
\hline Química, $\gamma_{04}$ & & & $-0.23 *$ & 0.11 \\
\hline Reto, $\gamma_{10}$ & $0.09 *$ & 0.03 & $0.12 \dagger$ & 0.07 \\
\hline Género, $\gamma_{11}$ & & & $-0.12 *$ & 0.06 \\
\hline Educación Ambiental, $\gamma_{11}$ & & & 0.03 & 0.08 \\
\hline Biología, $\gamma_{12}$ & & & 0.01 & 0.08 \\
\hline Quimica, $\gamma_{13}$ & & & 0.06 & 0.09 \\
\hline Habilidad, $\gamma_{20}$ & $0.32 * *$ & 0.03 & $0.49 * *$ & 0.07 \\
\hline Género, $\gamma_{21}$ & & & 0.01 & 0.07 \\
\hline Educación Ambiental, $\gamma_{22}$ & & & $-0.26^{*}$ & 0.1 \\
\hline Biologia, $\gamma_{23}$ & & & $-0.26^{*}$ & 0.1 \\
\hline Quimica, $\gamma_{24}$ & & & -0.11 & 0.1 \\
\hline Relevancia, $\gamma_{30}$ & $0.23^{* *}$ & 0.03 & $0.21^{*}$ & 0.07 \\
\hline Género, $\gamma_{31}$ & & & 0.07 & 0.05 \\
\hline Educación Ambiental, $\gamma_{32}$ & & & 0.08 & 0.09 \\
\hline Biologia, $\gamma_{33}$ & & & 0.05 & 0.09 \\
\hline Quimica, $\gamma_{34}$ & & & -0.11 & 0.08 \\
\hline \multicolumn{5}{|l|}{ Efectos Aleatorios } \\
\hline Varianza de nivel I, $r$ & 0.278 & & 0.279 & \\
\hline Reto, $\mathrm{u}_{1}$ & $0.027 * *$ & & $0.031 * *$ & \\
\hline Habilidad, $\mathrm{u}_{2}$ & $0.039 *$ & & $0.042 *$ & \\
\hline Relevancia, $\mathrm{u}_{3}$ & $0.021 *$ & & $0.015^{*}$ & \\
\hline Intercepto, $\mathrm{u}_{0}$ & $0.099 * *$ & & $0.082 * *$ & \\
\hline
\end{tabular}

Nota. $N=88$ estudiantes, 661 respuestas, $\dagger p<$ $0.1, * p<0.05, * * p<0.001$, se reportan efectos fijos con error estándar robusto; los predictores se centraron alrededor de la media. Modelo 1: Estimación de confiabilidad para el intercepto (compromiso cognitivo y afectivo) $=0.351$; para reto $=0.19$; para habilidad $=0.236$; para relevancia $=0.131$. Modelo 2: Estimación de confiabilidad para el intercepto (compromiso cognitivo y afectivo) $=0.317$; para reto $=0.188$; para habilidad $=0.25$; para relevancia $=0.097$.

La evaluación de la Hipótesis 4, el compromiso cognitivo y afectivo predice el rendimiento académico en Ciencias, fue realizada partir de un análisis de regresión lineal bivariado. El compromiso cognitivo y afectivo se usó como variable independiente mientras que la variable dependiente corresponde a las calificaciones finales de los estudiantes. El compromiso cognitivo y afectivo predice el desempeño en Ciencias $\mathrm{B}=0.28, F(1,80)=5.88, p<0.05 ; r=$ $0.26 ; R^{2}=0.07$. El resultado indica que cuando el compromiso cognitivo y afectivo incrementa una unidad, el desempeño en Ciencias aumenta 0.28 unidades.

\section{Discusión}

Los resultados del modelo nulo en esta investigación mostraron el compromiso como un fenómeno variable a largo de las clases de Ciencias. Es decir, el compromiso cognitivo y afectivo se mostró significativamente variable a lo largo de las unidades temáticas observadas. Este hallazgo es importante para el estudio de la motivación porque refuerza la idea de que esta, y en este caso particular el compromiso cognitivo y afectivo, debe estudiarse como un fenómeno dinámico, flexible, dependiente de las percepciones de los estudiantes frente a las tareas y no como una característica fija que los ubicaría como comprometidos o no con las clases de Ciencias en general (Schmidt, Rosenberg, \& Beymer, 2017). Las implicaciones educativas de esta idea sugieren que tanto estudiantes como docentes deben ser sensibles a dichos cambios, y que por tanto los docentes no deberían estar predispuestos ante ciertos alumnos que podrían mostrarse poco comprometidos en algunas clases de Ciencias y clasificarlos como no comprometidos (Smith \& Wilhelm, 2002); por su parte, los estudiantes también podrían ser más abiertos y tolerantes con ciertas temáticas y materias donde en algún momento pudieran percibir poco compromiso, pues en las siguientes clases, de acuerdo con las características de las actividades propuestas y las percepciones de reto, habilidad e importancia que atribuyan a las tareas, su compromiso puede variar y encontrar interesante un tema o tarea que inicialmente se consideró aburrida o demasiado difícil. Haciendo una analogía con la idea de mentalidad fija ("Yo no soy bueno para la Química") vs. mentalidad cambiante ("Con suficiente esfuerzo y dedicación puedo ser bueno para Química o para cualquier materia" (Dweck, 2006 citado por Shumow \& Schmidt, 2014) podrían describirse dos maneras de entender y estudiar el compromiso cognitivo y afectivo: como un rasgo fijo ("No tengo motivación para las Ciencias") versus el compromiso como un fenómeno cambiante ("Podría estar motivado para las Ciencias de acuerdo con las características de las tareas y mi percepción frente a ellas") y abogar por la 
idea del compromiso como fenómeno emergente, que depende tanto de factores fenomenológicos (percepciones de los estudiantes) como de factores instruccionales, y por tanto, apostarle a crear situaciones educativas que promuevan la emergencia del compromiso cognitivo y afectivo.

En este orden de ideas, se plantea como pertinente el desarrollo de intervenciones que desde la psicología educativa hagan énfasis en el reconocimiento del carácter dinámico de las percepciones de estudiantes y profesores alrededor del fenómeno del compromiso y su interacción con variables contextuales, de tal manera que se puedan diseñar contextos educativos ajustables y cambiantes en el tiempo, que respondan adaptativamente a las necesidades de los actores educativos, tal como se recomienda en recientes estudios sobre el tema (Schmidt et al., 2017; Strati et al., 2017).

En relación con la primera hipótesis de investigación, los resultados apoyan la hipótesis de que existe una relación significativa positiva entre percepciones de habilidad, reto, relevancia y compromiso cognitivo y afectivo de los estudiantes durante las clases de Ciencias. En relación con el reto y la habilidad como predictores del compromiso cognitivo y afectivo, resultados similares reportan Fullagar et al. (2013), Nakamura y Csikszentmihalyi (2002), Ochoa-Angrino (2012), Strati et al. (2017) y Shernoff et al. (2016).

Los resultados de las investigaciones previas señalan la necesidad de que los docentes durante las clases de Ciencias tomen en cuenta de manera integral estos tres factores. En primer lugar, las habilidades que los estudiantes tienen para resolver las tareas propuestas durante las clases y su percepción sobre dichas habilidades. Esta idea remite a la relevancia del conocimiento previo y su papel en los procesos de aprendizaje y de compromiso con el aprendizaje. En segundo lugar, identificar qué nuevos aprendizajes son posibles de acuerdo a ese nivel de habilidad y proponer prácticas educativas que permitan la construcción de esos nuevos aprendizajes. Es decir, prácticas educativas que sean percibidas como retadoras para el estudiante. Al respecto, Tomlinson y Strickland (2005) señalan: "[...] un estudiante solamente aprende cuando el trabajo es moderadamente retador y cuando tiene el apoyo necesario para realizar lo que inicialmente parece fuera de su alcance" (p. 163). Estos resultados siguen aportando evidencia a la idea que los estudiantes prefieren tareas retadoras en lugar de tareas fáciles (Shernoff \& Schmidt, 2008; Shernoff et al., 2016; Strati et al., 2017).

En relación con la percepción de relevancia acerca de las tareas que realizan en el salón de clases, esta investigación muestra que cuanta más relevancia encuentran los estudiantes en las tareas de Ciencias, mayor concentración, disfrute, sensación de éxito y bienestar experimentan; investigaciones previas relacionan la relevancia en la persistencia en tareas de Ciencias con la utilización de estrategias complejas para aprender y con la dedicación de mayor de tiempo a las tareas académicas (Assor, Kaplan, \& Roth, 2002; Canning \& Harackiewicz, 2015; Eccles, 2005; Hulleman et al., 2010; Koballa \& Glynn, 2007). Vale la pena mencionar que el compromiso fue similar para todos los estudiantes sin importar el tipo de curso ni el género. Parece haber un acuerdo implícito en todos los estudiantes que la relevancia de la tarea se relaciona con el compromiso. Los resultados en relación con esta última, sugieren a los educadores que además de contar con las habilidades de los estudiantes y ofrecer tareas retadoras, estas deben tener algún sentido personal, comunitario o social para ellos.

En relación con la segunda hipótesis, los resultados la apoyan parcialmente, pues no se encontraron diferencias significativas en compromiso de acuerdo al género, pero si algunas diferencias en relación con la asignatura. En esta línea, los estudiantes de Física mostraron mayor nivel de compromiso cognitivo y afectivo en comparación con los estudiantes de Química. Sin embargo, no hubo diferencias en las relaciones entre percepción de reto, habilidad e importancia entre estos dos grupos. Aspectos relacionados con las prácticas instruccionales podrían contribuir a explicar las diferencias; por ejemplo, que las clases magistrales predominaron en Química mientras prácticas más activas (experimentos, búsqueda de información, etc.) 
se presentaron en el curso de Física (véanse detalles en Pérez-Canto, 2016). Igualmente, cabe señalar que los estudiantes de Química obtuvieron los puntajes menores en habilidad, reto e importancia.

La tercera hipótesis de investigación predecía que las relaciones entre compromiso cognitivo y afectivo y percepciones de habilidad, reto y relevancia estaban moderadas por tipo de asignatura y género. Los resultados muestran un apoyo parcial a dicha hipótesis. En relación con el género como moderador de las relaciones entre percepción de habilidad, reto y relevancia, se encuentra que el género modera la relación entre reto y compromiso para las mujeres, quienes reportan menor nivel de compromiso que los hombres cuando perciben que las actividades son retadoras. Estos resultados son similares a los de Schmidt et al. (2010) y Strati et al., (2017), y podrían explicarse por el estereotipo de género que se señalaba en la parte de revisión. De acuerdo a este estereotipo, las mujeres no se consideran buenas para las Ciencias y si las actividades son retadoras tenderán a percibir menos compromiso que los chicos, quizá porque experimentan mayor ansiedad.

Igualmente, cuando los estudiantes de Física percibían mayor habilidad tendían a reportar mayor compromiso que los de Educación Ambiental y Biología. Como han reportado en estudios previos (Csikszentmihalyi \& Csikszentmihalyi, 1988) la habilidad se relaciona con el compromiso en la medida en que hay situaciones retadoras, si los estudiantes se sienten demasiado hábiles y las tareas son fáciles, tienden a reportar aburrimiento. En este sentido, las diferencias entre los grupos de Física, Educación Ambiental y Biología podrían explicarse porque el profesor de esta última asignatura se diferenciaba cualitativamente de los otros docentes en el tipo de prácticas que usó durante las clases: lecturas fuera del salón de clase, experimentos sobre circuitos eléctricos, exposición de los experimentos y mayor trabajo en grupo, comparado con los profesores de las otras dos asignaturas quienes tendían a dar clases magistrales, según resultados reportados por Pérez-Canto (2016) al estudiar las prácticas pedagógicas de estos docentes.

La cuarta hipótesis indagaba por la relación entre el compromiso cognitivo y afectivo y el desempeño académico. La relación significativa positiva entre compromiso y desempeño académico también fue hallada en estudios de Csikszentmihalyi et al. (2005), Larson (1988), Nakamura (1988) y Nakamura y Csikszentmihalyi (2002). Sin embargo, el tamaño del efecto no es alto, y podría explicarse porque los niveles de compromiso no fueron altos en la muestra observada y porque la medida de desempeño fue la calificación final del curso. En futuros estudios debería revisarse la relación entre compromiso y desempeño relacionado con la misma unidad temática observada.

El tipo de abordaje metodológico usado en este estudio, permite conocer las percepciones de las experiencias de los estudiantes en tiempo real durante las clases y también dar cuenta de su variabilidad en el tiempo. Esta información es relevante en términos de la literatura que apoya la idea de que la motivación es un fenómeno emergente, dinámico e interactivo, producto de la relación entre factores individuales y contextuales y, por tanto, susceptible de ser cambiada a partir de intervenciones educativas retadoras, significativas, que reconozcan, utilicen y transformen las habilidades de los estudiantes en tareas llenas de sentido. Con este tipo de abordaje metodológico, se puede hacer seguimiento a las relaciones entre eventos académicos (laboratorios, clases magistrales, salidas de campo, entre otros), situaciones particulares dentro del evento educativo (diferentes momentos en la realización de una práctica de laboratorio) y su relación con el compromiso, lo cual crea una descripción dinámica de la motivación de estudiantes y profesores, durante las actividades académicas.

Se debe mencionar que los resultados presentan una generalización limitada y se suscriben a escuelas colombianas, por lo que se hace pertinente la ampliación de la muestra y de los contextos observados. Una segunda limitación del estudio es que no se establecen relaciones entre las percepciones de compromiso, 
habilidad, relevancia y reto y el tipo de prácticas educativas que los docentes usan. Nuevos estudios podrían enfocarse en encontrar dichas relaciones; por ejemplo, analizando en qué medida la calidad de las prácticas docentes están relacionadas con el compromiso cognitivo y afectivo en las clases de Ciencias, tal como recientemente lo hacen Shernoff et al. (2016) y Strati et al. (2017). Estudios de este tipo podrían complementarse con aproximaciones cualitativas que enriquezcan la descripción sobre los cambios de compromiso cognitivo y afectivo y las percepciones de los estudiantes sobre el reto, la habilidad y la relevancia en función de las actividades específicas que realizan.

\section{Referencias}

Assor, A., Kaplan, H., \& Roth, G. (2002). Choice is good, but relevance is excellent: Autonomy-enhancing and suppressing teacher behaviors predicting students' engagement in schoolwork. British Journal of Educational Psychology, 72(2), 261-278. https://doi.org/10.1348/00070990 2158883

Canning, E. A., \& Harackiewicz, J. M. (2015). Teach it, don't preach it: The differential effects of directly-communicated and self-generated utility-value information. Motivation Science, 1(1), 41-71. https://doi. org/10.1037/mot0000015

Carmines, E., \& Mclver, J. (1981). Analyzing models with unobserved variables: Analysis of covariance structures. En G. Bohrnstedt \& E. Borgatta (Eds.), Social measurement: Current issues (pp. 65-115). Beverly Hills, CA: Sage.

Csikszentmihalyi, M. (2008). Flow: The psychology of optimal experience. Nueva York: Harper Perennial Modern Classics.

Csikszentmihalyi, M., \& Csikszentmihalyi, I. S. (1988). Introduction to Part IV. En M. Csikszentmihalyi \& I. S. Csikszentmihalyi (Eds.), Optimal experience: Psychological studies of flow in consciousness (pp. 251-265).
Cambridge, UK: Cambridge University Press.

Csikszentmihalyi, M., \& Larson, R. (1987). Validity and reliability of the ExperienceSampling Method. The Journal of Nervous and Mental Disease, 175(9), 526-536. https://doi.org/10.1097/00005053 $-198709000-00004$

Csikszentmihalyi, M., Abuhamdeh, S., \& Nakamura, J. (2005). Flow. En A. J. Elliot \& C. S. Dweck (Eds.), Handbook of competence and motivation (pp. 598-608). Nueva York: The Gilford Press.

Eccles, J. (2005). Subjective task value and the Eccles et al. Model of AchievementRelated Choices. En A. J. Elliot \& C. S. Dweck (Eds.), Handbook of competence and motivation (pp. 105-121). Nueva York: The Guilford Press.

Fleeson, W. (2007). Using experience sampling and multilevel modeling to study personsituation interactionist approaches to positive psychology. En A. D. Ong \& M. H. M. Van Dulmen (Eds.), Handbook of methods in positive psychology (pp. 501-514). Oxford, UK: Oxford University Press.

Fullagar, C. J., Knight, P. A., \& Sovern, H. S. (2013). Challenge/skill balance, flow, and performance anxiety. Applied Psychology, 62 (2), 236-259. https://doi.org/10.1111/j.1 464-0597.2012.00494.x

Furrer, C., Skinner, E., \& Pitzer, J. (2014). The influence of teacher and peer relationships on students' classroom engagement and everyday motivational resilience. National Society for the Study of Education, 113(1), 101-123. https://doi.org/10.3102/00346543 08325583

Hektner, J. M., Schmidt, J. A., \& Csikszentmihalyi, M. (2007). Experience sampling method: Measuring the quality of everyday life. Thousand Oaks, CA: Sage.

Hill, L. G., \& Werner, N. E. (2006). Affiliative motivation, school attachment, and aggression in school. Psychology in the Schools, 43, 231-246. https://doi.org/10.100 2/pits.20140 
Hulleman, C. S., Godes, O., Hendricks, B. L., \& Harackiewicz, J. M. (2010). Enhancing interest and performance with a utility value intervention. Journal of Educational Psychology, 102, 880-895. https://doi.org/10 $.1037 / \mathrm{a} 0019506$

Koballa, T. R., \& Glynn, S. M. (2007). Attitudinal and motivational constructs in science learning. En S. Abell \& N. Lederman (Eds.), Handbook on science education (pp. 75-124). Mahwah, NJ: Erlbaum.

Lam, S-f., Jimerson, S., Wong, B. P. H., Kikas, E., Shin, H., Veiga, F. H. ... Zollneritsch, J. (2014). Understanding and measuring student engagement in school: The results of an international study from 12 countries. School Psychology Quarterly, 29, 213-232. ht tps://doi.org/10.1037/spq0000057

Larson, R. W. (1988). Flow and writing. En M. Csikszentmihalyi \& I. S. Csikszentmihalyi (Eds.), Optimal experience: Psychological studies of flow in consciousness (pp. 150-171). Cambridge, UK: Cambridge University Press.

Leech, N. L., Barrett, K. C., \& Morgan, G. A. (2008). SPSS for intermediate statistics. Use and interpretation. Mahwah, NJ: Laurence Erlbaum.

Lloret-Segura, S., Ferreres-Traver, A., Hernández-Baeza, A., \& Tomás-Marco, I. (2014). El análisis factorial exploratorio de los ítems: una guía práctica, revisada y actualizada. Anales de Psicología, 30(3), 1151-1169. https://doi.org/10.6018/analesp s.30.3.199361

McDonald, R. P., \& Ho, M. R. (2002). Principles and practice in reporting structural equation analyses. Psychological Methods, 7(1), 64-82. https://doi.org/10.1037//1082989X.7.1.64

Nakamura, J. (1988). Optimal experience and the uses of talent. En M. Csikszentmihalyi \& I. S. Csikszentmihalyi (Eds.), Optimal experience: Psychological studies of flow in consciousness (pp. 319-326). Cambridge, UK: Cambridge University Press.
Nakamura, J., \& Csikszentmihalyi, M. (2002). The concept of flow. En C. R. Snyder \& S. J. López (Eds.), Handbook of positive psychology (pp. 89-105). Nueva York: New York University Press.

Netemeyer, R., Bearden, W., \& Sharma, S. (2003). Scaling procedures: Issues and applications. Thousand Oaks, CA: Sage.

Ochoa-Angrino, S. (2012). Factors related to students' engaged reading in high school science classrooms (Tesis doctoral, Northern Illinois University). Recuperada de http://gradwor ks.umi.com/35/13/3513148.html

Patrick, H., Ryan, A. M., \& Kaplan, A. (2007). Early adolescents' perceptions of the classroom social environment, motivational beliefs, and engagement. Journal of Educational Psychology, 99(1), 83-98. https://doi.org/10.1037/0022-0663. 99.1 .83

Pérez-Canto. G (2016). El apoyo emocional en los procesos de enseñanza y aprendizaje en clases de Ciencias (Tesis de pregrado). Pontificia Universidad Javeriana, Cali, Colombia.

Raudenbush, S. W., Bryk, A. S., \& Congdon, R. (2010). HLM 7 for Windows [Software de computación]. Lincolnwood, IL: Scientific Software International, Inc.

Raudenbush, S. W., \& Bryk, A. S. (2002). Hierarchical linear models: Applications and data analysis methods (2.nd ed.). Newbury Park, CA: Sage.

Reyes, M. R., Brackett, M. A., Rivers, S. E., White, M., \& Salovey, P. (2012). Classroom emotional climate, student engagement, and academic achievement. Journal of Educational Psychology, 104(3), 700-712. ht tps://doi.org/10.1037/a0027268

Rigo, D. (2017). Docentes, tareas y alumnos en la definición del compromiso. Investigando el aula de nivel primario de educación. Educação em Revista, 33, 1-24. https://doi.o $\mathrm{rg} / 10.1590 / 0102-4698154275$

Rozek, C. S., Hyde, J. S., Svoboda, R. C., Hulleman, C. S., \& Harackiewicz, J. M. (2015). Gender differences in the effects of a utility-value intervention to help parents motivate adolescents in mathematics and 
science. Journal of Educational Psychology, 107, 195-206. https://doi.org/10.1037/a003 6981

Schmidt, J. A., Rosenberg, J. M., \& Beymer, P. N. (2017). A person-in-context approach to student engagement in science: Examining learning activities and choice. Journal of Research in Science Teaching, 55(1), 19-43. h ttps://doi.org/10.1002/tea.21409

Schmidt, J. A., \& Smith, M. C. (Agosto, 2008). Looking inside high school classrooms: An exploration of males' and females' subjective experience. Grant proposal funded by the National Science Foundation, Research on Gender in Science Education program (N. ${ }^{\circ}$ HRD-0827526). Washington, DC.

Schmidt, J. A., Shumow, L. \& Zaleski, D. (abril, 2012). Multiple perspectives on student motivation, learning, and engagement in high school biology labs. Trabajo presentado en el Annual Meeting of the American Educational Research Association, Vancouver, British Columbia, Canada.

Schmidt, J. A., Strati, A. D., \& Kackar, H. Z. (marzo, 2010). Similar and different: comparisons of males' and females' achievement, attitudes, interest, and experience in high school science. Póster presentado en el Biennial Meeting of the Society for Research on Adolescence, Philadelphia, PA.

Shernoff, D. J. (2010). Engagement in afterschool programs as a predictor of social competence and academic performance. American Journal of Community Psychology, 45, 325-337. https://doi.org/10.1007/s1046 4-010-9314-0

Shernoff, D. J. (2012). Engagement and positive youth development: Creating optimal learning environments. En K. R. Harris, S. Graham \& T. Urdan (Eds.), The APA educational psychology handbook (Vol. 3, pp. 195-220) Washington, DC: American Psychological Association.

Shernoff, D. J., \& Anderson, B. (2013). Flow and optimal learning environments. En J. Froh \& A. Parks-Sheiner (Eds.), Activities for teaching positive psychology: A guide for instructors (pp. 109-115). Washington, DC: The American Psychological Association.

Shernoff, D. J., \& Bempechat, J. (Eds.). (2014). Engaging youth in schools: Evidence-based models to guide future innovations. Nueva York: NSSE Yearbook by Teachers College Record.

Shernoff, D. J., \& Schmidt, J. A. (2008). Further evidence of an engagement-achievement paradox among U.S. high school students. Journal of Youth and Adolescence, 37, 564-580. https://doi.org/10.1007/s10964-0 07-9241-z

Shernoff, D. J., \& Vandell, D. L. (2007). Engagement in after-school program activities: Quality of experience from the perspective of participants. Journal of Youth and Adolescence, 36, 891-903. https://doi.or $\mathrm{g} / 10.1007 / \mathrm{s} 10964-007-9183-5$

Shernoff, D. J., Abdi, B., Anderson, B., \& Csikszentmihalyi, M. (2014). Flow in schools revisited: Cultivating engaged learners and optimal learning environments. En M. Furlong, R. Gilman $\&$ S. Heubner (Eds.), Handbook of positive psychology in the schools (pp. 211-226). NuevaYork: Routledge.

Shernoff, D. J., Csikszentmihalyi, M., Schneider, B., \& Shernoff, E. S. (2003). Student engagement in high school classrooms from the perspective of flow theory. School Psychology Quarterly, 18, 158-76. https://do i.org/10.1521/scpq.18.2.158.21860

Shernoff, D. J., Kelly, S., Tonks, S., Anderson, B., Cavanagh, R., ..., \& Abdi, B. (2016). Student engagement as a function of environmental complexity in high school classrooms. Learning and Instruction. doi: https://doi.org/10.1016/j.learninstruc.2 015.12.003.

Shukla, K., Konold, T., \& Cornell, D. (junio, 2015). School climate and student engagement: A concurrent validity investigation through a multilevel multivariate approach. Trabajo presentado en el Annual Meeting of the American Educational Research Association, Chicago, USA. 
Shumow, L., \& Schmidt, J. A. (2014). Enhancing adolescents' motivation for science. Thousand Oaks, CA: Corwin Press.

Skinner, E. A., \& Belmont, M. J. (1993). Motivation in the classroom: Reciprocal effect of teacher behavior and student engagement across the school year. Journal of Educational Psychology, 85, 571-581. http s://doi.org/10.1037/0022-0663.85.4.571

Skinner, E. A., Furrer, C., Marchand, G., \& Kindermann, T. (2008). Engagement and disaffection in the classroom: Part of a larger motivational dynamic? Journal of Educational Psychology, 100, 765-781. https ://doi.org/10.1037/a0012840

Skinner, E. A., Kindermann, T. A., \& Furrer, C. J. (2009). A motivational perspective on engagement and disaffection: Conceptualization and assessment of children's behavioral and emotional participation in academic activities in the classroom. Educational and Psychological Measurement, 69, 493-525. https://doi.org/1 $0.1177 / 0013164408323233$

Smith, M. W., \& Wilhelm, J. D. (2002). "Reading don't fix no Chevys": Literacy in the lives of young men. Portsmouth, NH: Heinemann Publishing.

Steinberg, L., Brown, B., \& Dornbush, S. (1996). Beyond classroom: Why school reform has failed and what parents need to do. Nueva York: Simon \& Schuster.

Strati, A. D., Schmidt, J. A., \& Maier, K. S. (2017). Perceived challenge, teacher support, and teacher obstruction as predictors of student engagement. Journal of Educational Psychology, 109(1), 131-147. ht tps://doi.org/10.1037/edu0000108

Tomlinson, C. A., \& Strickland, C. A. (2005). Differentiation in practice: A resource guide for differentiating curriculum, grades 9-12. Alexandria, VA: Association for Supervision and Curriculum Development.

Vansteenkiste, M., Simons, J., Lens, W., Sheldon, K. M., \& Deci, E. L. (2004). Motivating learning, performance, and persistence: The synergistic effects of intrinsic goal contents and autonomy- supportive contexts. Journal of Personality and Social Psychology, 87, 246-260. https://d oi.org/10.1037/0022-3514.87.2.246

Velicer, W. F., Eaton, C. A., \& Fava, J. L. (2000). Construct explication through factor or component analysis: A review and evaluation of alter-native procedures for determining the number of factors or components. En R. D. Goffin \& E. Helmes (Eds.), Problems and solutions in human assessment: Honoring Douglas N. Jackson at seventy (pp. 41-71). Norwell, MA: Kluwer Academic.

Vitiello, V. E., Booren, L. M., Downer, J. T., \& Williford, A. P. (2012). Variation in children's classroom engagement throughout a day in preschool: Relations to classroom and child factors. Early Childhood Research Quarterly, 27, 210-220. https://doi .org/10.1016/j.ecresq.2011.08.005

Wang, Z., Bergin, C., \& Bergin, D. A. (2014). Measuring engagement in fourth to twelfth grade classrooms: The classroom engagement inventory. School Psychology Quarterly, 29(4), 517-535. https://doi.org/1 $0.1037 / \mathrm{spq} 0000050$

Zuzanek, J. (mayo, 1999). Experience sampling method: Current and potential research applications. Trabajo presentado en el Workshop on Time-Use Measurement and Research, National Research Council, Washington, DC.

\section{Apéndice 1}

\footnotetext{
Modelo No condicionado Nivel I

$\mathrm{Y}_{\mathrm{ij}}($ Compromiso cognitivo y afectivo $)=\beta_{0 \mathrm{j}}+$

Modelo Nivel II

$\beta_{0 j}=\gamma_{00}+u_{0 j}$

Donde:

$\mathrm{Y}_{\mathrm{ij}}$ es la variable dependiente, la cual mide el compromiso cognitivo y afectivo de los estudiantes bajo estudio; representa el puntaje promedio de un estudiante i en un momento particular j.
} $r_{\mathrm{ij}}$ 
$\beta_{0 j}$ es el promedio de compromiso para el estudiante i cuando la variable independiente $\mathrm{X}$ (habilidad, reto, relevancia) es cero. Esta variable se relaciona con $\mathrm{Y}_{\mathrm{ij}}$ a través de la siguiente ecuación: $\beta_{0 j}=\gamma_{00}+u_{0 j}$,

$\mathrm{r}_{\mathrm{ij}}$ es la desviación del promedio de un estudiante $i$ en un tiempo $j$ con respecto a la media del grupo. Incluye el impacto de todas las variables no observables o no medibles en esta investigación.

$\gamma_{00}$ es el intercepto, el cual representa el promedio de compromiso para todos los participantes.

$\mathrm{u}_{0 \mathrm{j}}$ es la variabilidad del estudiante respecto a la media del grupo o el efecto único de cada participante en el compromiso durante las clases. Recoge factores específicos o únicos de cada estudiante no medibles a través de las otras variables.

\section{Modelo Condicional 1}

\section{Modelo Nivel I}

$\mathrm{Y}_{\mathrm{ij}}($ Compromiso $)=\beta_{0 \mathrm{j}}+\beta_{1 \mathrm{j}} *\left(\right.$ Reto $\left._{\mathrm{ij}}\right)+$ $\beta_{2 j} *\left(\right.$ Habilidad $\left._{i j}\right)+\beta_{3 j} *\left(\right.$ Relevancia $\left._{i j}\right)+r_{i j}$

\section{Modelo Nivel II}

$$
\begin{aligned}
& \beta_{0 \mathrm{j}}=\gamma 00+\mathrm{u}_{0 \mathrm{j}} \\
& \beta_{1 \mathrm{j}}=\gamma_{10}+\mathrm{u}_{1 \mathrm{j}} \\
& \beta_{2 \mathrm{j}}=\gamma_{20}+\mathrm{u}_{2 \mathrm{j}} \\
& \beta_{3 \mathrm{j}}=\gamma_{30}+\mathrm{u}_{3 \mathrm{j}}
\end{aligned}
$$

$\mathrm{Y}_{\mathrm{ij}}$ representa la puntuación de compromiso cognitivo y afectivo que presenta un estudiante $\mathrm{i}$ en el momento $\mathrm{j}$ de la clase de Ciencias.

$\beta_{0 j}$ es el promedio de compromiso cognitivo y afectivo de un estudiante i a través del tiempo sin tener en cuenta el efecto de las demás variables (o suponiendo que son cero).

$\beta_{1 j}$ es el coeficiente de regresión del índice de la fuerza de la asociación entre la percepción de reto y el compromiso para un estudiante i cuyos valores en la percepción de habilidad y de relevancia se encuentran dentro del promedio. Representa el incremento en la variable compromiso cada unidad en reto.

$\beta_{2 j}$ es el coeficiente de regresión del índice de la fuerza de la asociación entre la percepción de habilidad y compromiso para un estudiante i cuyos valores en la percepción de reto y de relevancia de la actividad se ubican en el promedio. Representa el incremento en la variable compromiso por cada unidad en habilidad.

$\beta_{3 j}$ es el coeficiente de regresión del índice de la fuerza de la asociación entre la percepción de relevancia de la tarea y el compromiso para un estudiante i cuyos valores en la percepción de habilidad y reto se encuentran en el promedio. Representa el incremento en compromiso por cada unidad en relevancia.

$\mathrm{r}_{\mathrm{ij}}$ representa la variabilidad del puntaje del estudiante i respecto a la media del grupo.

En el nivel 2 del modelo:

$\gamma_{00}$ es la media de compromiso para todos los estudiantes.

$\mathrm{u}_{0 \mathrm{j}}$ es el efecto único de cada estudiante en el compromiso cognitivo y afectivo.

$\gamma_{10}$ es el promedio de la percepción de reto para todos los estudiantes,

$\mathrm{u}_{1 \mathrm{j}}$ es el efecto único de cada estudiante en la percepción de reto;

$\gamma_{20}$ es el promedio de la percepción de habilidad de los estudiantes.

$\mathrm{u}_{2 \mathrm{j}}$ es el efecto único de cada estudiante en la percepción de habilidad.

$\gamma_{30}$ es el promedio de la percepción de relevancia para todos los estudiantes.

$\mathrm{u}_{3 \mathrm{j}}$ es el efecto único de cada estudiante en la percepción de relevancia.

\section{Notas}

1 Donde 1 correspondía a la pertenencia a determinado grupo y 0 la no pertenencia. Por ejemplo, si un estudiante pertenecía a Física se marcaba 1 y a los demás se les marcaba 0 ; si era mujer 1 y hombre 0 .

* Artículo de investigación. Este artículo es producto del proyecto de investigación "Compromiso con la Lectura y Aprendizaje 
Solanlly Ochoa-Angrino, Jairo A. Montes-González, Tatiana Rojas-Ospina.

de las Ciencias Naturales (Código 3976)"

financiado por la Pontificia Universidad Javeriana Cali, Oficina de Investigación, Desarrollo e Innovación. 